
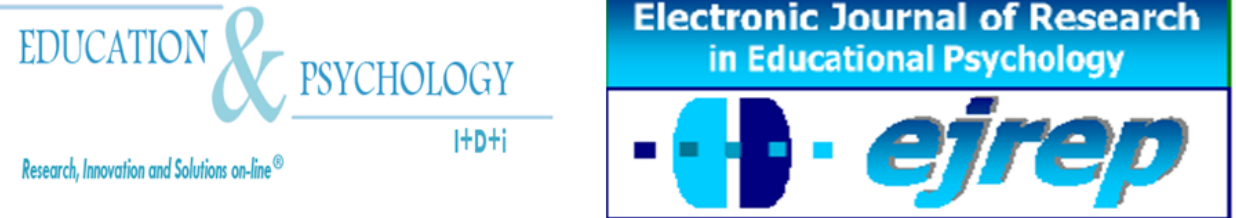

Editorial EOS

\title{
Estudio del maltrato psicológico, en las relaciones de pareja, en jóvenes universitarios
}

\section{Macarena Blázquez Alonso ${ }^{1}$, Juan Manuel Moreno Man- so $^{2}$, María Elena García-Baamonde Sánchez ${ }^{3}$}

${ }^{1}$ Departamento de Psicología, Universidad de Extremadura, Cáceres

${ }^{2}$ Departamento de Psicología, Universidad de Extremadura, Badajoz

${ }^{3}$ Departamento de Psicología, Universidad de Extremadura, Badajoz

\section{España}

Correspondencia: Macarena Blázquez Alonso. Universidad de Extremadura, Av. de la Universidad, s/n. 10071 Cáceres. España. E-mail: mablazqueza@unex.es

(C) Education \& Psychology $\mathrm{I}+\mathrm{D}+\mathrm{i}$ and Editorial EOS (Spain) 


\section{Resumen}

Introducción. La mayoría de los estudios en torno a la violencia conyugal presentan un marcado interés por la dimensión física del maltrato, ignorando el maltrato psicológico a pesar de su elevada incidencia y graves repercusiones en la víctima.

Objetivo. El artículo tiene como propósito aportar un mayor conocimiento sobre el fenómeno del maltrato psicológico en las relaciones de noviazgo en jóvenes universitarios mediante la identificación de factores asociados al maltrato psicológico, así como la determinación del grado en que son afectados y la posible relación existente entre éstos y la variable edad.

Método. Participaron 216 estudiantes universitarios de diferentes titulaciones de la Facultad de Educación de la Universidad de Extremadura con edades comprendidas entre los 17 y 23 años, distribuidos en cuatro grupos diferenciados atendiendo a los siguientes intervalos de edad: 17-18, 19-20, 21-22 y 23 ó más. Se diseñó un cuestionario de maltrato emocional compuesto por los factores desvalorización, hostilidad, indiferencia, intimidación, imposición de conductas, culpabilización y bondad aparente, que posibilitó la recogida y posterior análisis de datos, en función de otras investigaciones de carácter nacional e internacional en materia de violencia coyugal.

Resultados. Los resultados indican que todos los componentes del maltrato psicológico analizados se encuentran afectados. Existen evidencias de un mayor repertorio conductual por parte de los jóvenes de 17-18 años frente al resto de intervalos de edad, en lo referido al maltrato psicológico en la pareja. Los datos muestran diferencias significativas entre los sujetos de 17-18 años y 21-22 en los indicadores: descalificaciones, trivializaciones, conductas destructivas, aislamiento social.

Discusión y conclusiones. Los resultados obtenidos, acordes a otras investigaciones realizadas permiten afirmar que los jóvenes universitarios con edades comprendidas entre los 17 y 23 años describen patrones de interacción coactiva en sus relaciones de noviazgo. Asimismo, se comprueba el empleo diferencial de los indicadores de violencia psicológica según la edad en que se encuentra el sujeto, siendo puestos en práctica en mayor medida por los jóvenes de 17-18 años. Se propone anticiparnos a las situaciones de conflictividad conyugal desde el contexto educativo mediante el entrenamiento en competencias emocionalmente "inteligentes".

Palabras Clave: maltrato psicológico, violencia en la pareja, noviazgo, universitarios, inteligencia emocional

Recibido: 09/03/09

Aceptación inicial: 13/03/09

Aceptación final: 15/04/09 


\title{
A study of psychological abuse in couple relationships in young university students
}

\begin{abstract}
Introduction. Most studies on domestic violence presents a mar-market interest in the physical abuse, psychological abuse ignored despite its high incidence and serious consequences for the victim.
\end{abstract}

Aim. The article aims to provide greater knowledge about the phenomenon of psychological abuse in dating relationships in college youth through the identification of factors associated with psychological abuse, as well as co-determining the degree to which they are affected and the possible relationship between them and the variable age.

Method. 216 students participated in different degrees of the Faculty of Education, University of Extremadura, aged between 17 and 23 years, divided into four distinct groups based on the following age intervals: 17-18, 19-20, 21 -- 22 and 23 or more. We designed a questionnaire consisting of emotional abuse depreciation factors, hostility, indifference, intimidation, imposition of behavior, scapegoating and apparent kindness that enabled the collection and subsequent analysis of data, according to other investigations of national and international level coyugal violence.

Results. The results indicate that all components of the psychological abuse analyzed are affected. There is evidence of a larger behavioral repertoire by young people of 17-18 years compared to other age ranges, as regards the psychological ill-treatment in couples. The data show significant differences between subjects of 17-18 years and 21-22 in the indicators: vetting, trivialized, de-constructive behavior, social isolation.

Discussion and conclusions. The results are consistent with other investigations carried out indicate that the university students aged between 17 and 23 years described coercive interaction patterns in their dating relationships. Also, there is differential use of the indicators of psychological violence as the age at which the subject is being implemented to a greater extent by young people of 17-18 years. It is proposed to anticipate situations of marital conflict from the con-text educational skills through training in emotionally intelligent.

Key words: psychology abuse, violence in the intimate partner, engagement, university, emotional intelligence

Received: 03/09/09 Initial Acceptance: 03/13/09 Definitive Acceptance: 04/15/09 


\section{Introducción}

La sociedad occidental está sujeta a constantes cambios sociales que inciden en la relación de pareja. De hecho, hoy cohabitan diversos patrones que configuran este tipo relación: noviazgo, matrimonio, parejas de hecho, parejas homosexuales, sujetos divorciados que conviven de nuevo con su excónyuge, etc. (Salazar, Torres y Rincón, 2005). A pesar de estas situaciones, cuando se trata de analizar el fenómeno de los malos tratos en la pareja, las posturas que se siguen adoptando resultan de lo más reduccionistas y estereotipadas. De esta forma, el interés por las manifestaciones de este tipo de violencia se restringe a la dimensión física del maltrato, adhiriéndose a modelos tradicionales con un esquema de pareja hombremujer, habitualmente, bajo el vínculo del matrimonio o una convivencia en común, donde las conductas de abuso de poder que representan los malos tratos, quedan atribuidas al hombre en concepto de su superioridad física. Sin embargo, la realidad nos muestra que ejercer control sobre la pareja es una cuestión que trasciende a la mera agresión física, cuya expresión en el seno del matrimonio es producto del incremento progresivo de patrones de interacción coactiva aparecidos en etapas precedentes a la consolidación de la pareja, es decir, durante el noviazgo (Corsi y Ferreira, 1998; Hirigoyen, 2006; O’Leary, 1988; Straus, 1983; Walker, 1979). Así, nos encontramos con nuevos argumentos que rompen con superficiales reduccionismos sociales que simplifican el campo de la violencia conyugal omitiendo una realidad también manifiesta en parejas de muy corta edad (Reiss y Roth, 1993).

Por esta razón, es necesario tener en cuenta la existencia de definiciones sobre la violencia íntima o "dating violence" que arrojan aspectos reveladores en este sentido. Tal es el caso de Sugarman y Hotaling (1989), quienes conceptualizan el fenómeno como "aquel que tiene lugar en el marco de una relación romántica mediante la realización directa de cualquier acto violento o conducta intimidatoria infligida por un miembro de una pareja no casada sobre el otro", introduciendo aspectos hasta momento pasados por alto. En primer lugar, destacan la presencia de conductas de agresión psicológica frente a las ya consabidas manifestaciones físicas de este fenómeno, advirtiendo una severa dificultad en su detección (Follingstad, Rutledge, Berg, Hause y Polek, 1990; O’Leary, 1999) debido a las huellas "invisibles" (Asensi, 2008) que generan en el psiquismo de la víctima, y que son originadas por su paulatino e insidioso (Loring, 1994) modo de operar. A su vez, y en segundo lugar, contribuye a la eliminación del tópico social que limita la existencia de los malos tratos en las relaciones íntimas a un fenómeno privativo de aquellas parejas que se encuentran bajo el vínculo conso- 
lidado del matrimonio. En esta línea, diversas investigaciones internacionales señalan que las cifras de prevalencia de violencia íntima en jóvenes fluctúa entre el 9 y 46\% (Glass, Freland, Campbell, Yonas, Sharp y Kub, 2003, Grumbaum, Kann, Kinchen, Williams, Ross, Lowry y Kolbel, 2002; Price, Byers, Sears, Whelan y Saint-Pierre, 2000) presentando considerables barreras de cara a su identificación por la existencia de aspectos carenciales o precarios en cuanto a la experiencia e información que en estos momentos posee el sujeto para valorar adecuadamente la situación de pareja que sostiene y retroalimenta (Bertalanffy, 1959).

En esta dirección, podemos afirmar que el retardo en dicha detección puede alcanzar trágicas consecuencias, si tenemos en cuenta la existencia de estudios (Smith, 1993) que destacan la tortura psicológica descrita por manifestaciones tales a la desvalorización, hostilidad, indiferencia, intimidación, imposición de conductas, culpabilización y bondad aparente (Taverniers, 2001) como el peor aspecto de los malos tratos (OMS, 1994). Una tesis que, es avalada por un amplio volumen de trabajos que coinciden en explicar la magnitud de la violencia psicológica con un impacto psicológico similar o superior al ocasionado por las agresiones físicas en la salud mental del sujeto que los experimenta (Henning y Klesges, 2003; Marshall, 1992; Sackett y Saunders, 1999; Street y Arias, 2001).

Recientemente, investigaciones comoBasoglu (2007) ha confirmado estos hallazgos aseverando que los sujetos víctimas de manipulación, humillaciones e intenso estrés psicológico, sufren años después, en términos de deterioro, las mismas consecuencias que aquellos sometidos a malos tratos físicos.

Es por ello que, optando por la clasificación elaborada por Taverniers sobre las manifestaciones y/o indicadores asociadas al maltrato psicológico/emocional (desvalorización, hostilidad, indiferencia, intimidación, imposición de conductas, culpabilización y bondad aparente), esta investigación pretende, identificar la presencia de cada una de ellas en las relaciones de noviazgo de estudiantes universitarios, y contribuir con dicho cometido a documentar y concienciar sobre esta gran desconocida forma de maltrato. A la vez que, responder al vacío conceptual existente en torno a este tipo de violencia, y buscar estrategias que favorezcan un cambio de actitudes, impidiendo la aparición de conductas de agresión psicológica, y permitiendo el desarrollo de una resolución de conflictos en la pareja sujeta a la confrontación intelectual y el pacto (Hué, 1994), entre otras, competencias comprendidas en el terreno de la inteligencia emocional. 
De esta forma, la presente investigación va dirigida a identificar los indicadores y/o manifestaciones de maltrato psicológico que se producen en aquellas etapas o momentos anteriores a lo que podemos entender como la consolidación del sistema conyugal (matrimonio, inicio de convivencia...) ya que, apenas existe constancia, a nivel nacional y en contraste con otros países, de trabajos que aborden estas situaciones de violencia en la pareja en este período, es decir, durante el noviazgo.

Finalmente, señalar que además de estos inconvenientes, existen más obstáculos inherentes a la naturaleza del maltrato emocional, cómo detectar el abuso psicológico y concretar los signos que determinan su existencia a través de instrumentos que proporcionen fieles testimonios del mismo. Este es un desafío que aún no ha sido asumido de forma precisa desde el ámbito asistencial destinado a las víctimas de violencia en pareja, constituyendo un último eslabón cimentado en la prioritaria y ardua labor, hoy por hoy inexistente, de informar, formar y sensibilizar a la población en general, sobre la naturaleza e implicaciones del abuso psicológico en la salud conyugal (Montero, 2005).

\section{Método}

\section{Participantes}

La investigación se desarrolla en el contexto de la Universidad de Extremadura con alumnos de edades comprendidas entre los 17 y 23 años matriculados en las siguientes titulaciones impartidas en la Facultad de Educación: maestro especialista en Audición y Lenguaje, Educación Especial, Educación Física, Educación Infantil, Educación Primaria, Lenguas Extranjeras y Psicopedagogía. Con la finalidad de obtener una muestra lo suficientemente representativa contactamos con la Secretaría del Centro para posteriormente pasar a realizar la selección muestral partiendo de la aplicación SPSS 15.0.

Participaron 216 estudiantes universitarios de primer a último curso con edades comprendidas entre los 17 y 23 años. De ellos, 157 son mujeres y 59 varones. En la investigación se ha considerado como objeto de análisis la muestra global de jóvenes $(n=216)$, sin grupo de control. Se establecieron cuatro grupos diferenciados atendiendo a los siguientes intervalos de edad: 17-18, 19-20, 21-22 y 23 ó más. Existe un mayor número de sujetos en el intervalo de edad que abarca los 21-22 años $(38,4 \%)$ mientras el intervalo de edad más pobre en sujetos es 
el que delimita los 23 años y edades superiores $(11,1 \%)$. La distribución de los jóvenes atendiendo a estos intervalos de edad queda recogida en la siguiente tabla:

Tabla 1. Porcentaje de casos atendiendo a los intervalos de edad

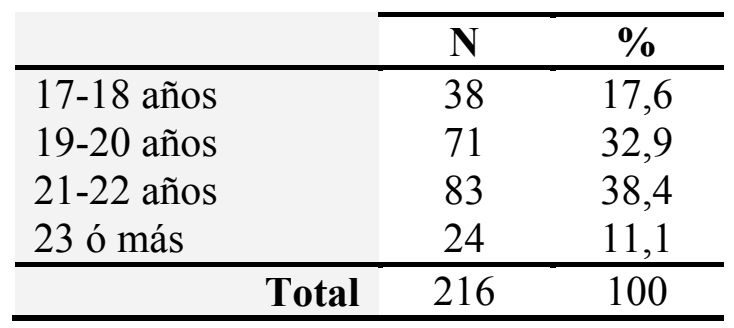

A continuación la tabla 2, muestra la distribución de los jóvenes atendiendo a la edad. Se refleja la cantidad de jóvenes por edad sin agrupar en intervalos. Comprobamos que los 21 años aparecen como la edad que presenta un mayor número de sujetos $(\mathrm{n}=44)$ frente a los 17 años $(\mathrm{n}=12)$ :

Tabla 2. Porcentaje de casos atendiendo a la edad

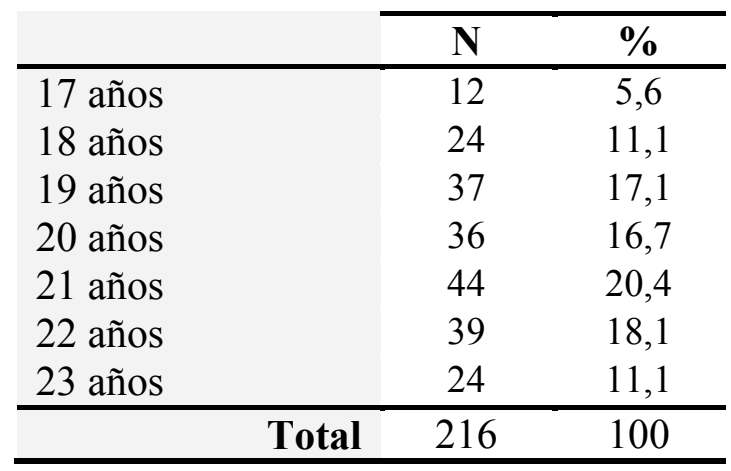

\section{Instrumentos}

Para evaluar el maltrato psicológico/emocional en las relaciones de noviazgo, diseñamos un Cuestionario de Maltrato Emocional (CME), basándonos para ello en varias encuestas nacionales e internacionales e instrumentos de cribado y diagnóstico de la violencia en la pareja. 
La ausencia de instrumentos originales validados para el cribado o el diagnóstico de la violencia en la pareja en España, hicieron necesario recurrir a algunas adaptaciones disponibles de escalas norteamericanas.

Hay varias escalas y cuestionarios que han alcanzado una enorme popularidad, como es el caso de las Conflict Tactics Scales (CTS) de Straus (1979) o el Index of Spouse Abuse (ISA) de Hudson y McIntosh (1981). Algunas de estas escalas, como la CTS, se han utilizado ampliamente en encuestas representativas de la población general, como es el caso de la US National Family Violence Survey o la Canadian Violence Against Women Survey. Estos instrumentos han sido extensamente empleados como instrumentos de cribado y de diagnóstico en el ámbito hospitalario como ayuda al personal sanitario en su tarea de detección y tratamiento de los casos de violencia en la pareja.

En nuestro caso, para el diseño del cuestionario nos basamos en los siguientes instrumentos:

- Abuse Disability Questionnaire (ADQ) de McNamara y Brooker (2000)

- Conflict Tactics Scale Revised (CTS2) de Straus, Hamby, Boney-McCoy y Sugarman (1996)

- Index of Spouse Abuse (ISA) de Hudson y McIntosh (1981)

- Inventario de evaluación del maltrato a la mujer por su pareja (APCM) de Matud, Caballeira y Marrero (2001)

- Non-Physical Abuse of Partner Scale (NPAPS) de Garner y Hudson (1992)

- Norvold Abuse Questionnaire (NOR-AQ) de Swahnberg y Wijma (2003)

- Partner Abuse Scale: Non-physical (PASNP) de Hudson (1990)

- Psychological Maltreatment Of Women Inventory (PMWI) de Tolman (1989)

- Psychological Violence Inventory de Sonkin (2001)

El cuestionario compuesto por 92 reactivos categóricos con formato gradual nos ha permitido estimar el riesgo de que existan manifestaciones de maltrato psicológico en cada uno de los 7 factores evaluados (ver Tabla 3). A su vez, nos informó de la tipología diferencial de conductas de agresión psicológica puestas en práctica en el sistema conyugal según el sexo del agresor. 


\section{Procedimiento}

En primer lugar, con la finalidad de obtener una muestra lo suficientemente representativa del alumnado universitario de las edades comprendidas entre los 17 y 23 años matriculados en la Facultad de Educación contactamos con la Secretaría del Centro. Posteriormente realizamos la selección muestral partiendo de la aplicación SPSS 15.0. A continuación, solicitamos la colaboración del profesorado del centro, al que dimos a conocer el contenido de la investigación. Posteriormente, elaboramos junto a los docentes un calendario para la aplicación del cuestionario de maltrato emocional.

La aplicación del cuestionario se realizó durante el curso académico 2007-2008. Se administró de forma colectiva, con una duración aproximada de 45 minutos. A continuación se codificaron las variables objeto de estudio en la matriz del SPSS y se introdujeron los datos obtenidos. Una vez introducidos los datos, llevamos a cabo el análisis de los datos, contrastando los datos obtenidos con los de otras investigaciones de carácter nacional e internacional en materia de violencia conyugal.

Tabla 3. Factores y subfactores del Cuestionario de Maltrato Emocional

\begin{tabular}{ll}
\hline \multicolumn{1}{c}{ FACTORES } & \multicolumn{1}{c}{ SUBFACTORES } \\
\hline & 1. Ridiculización \\
2. Descalificaciones \\
3. Trivializaciones \\
& $\begin{array}{l}\text { 4. Oposiciones } \\
\text { 5. Desprecio }\end{array}$ \\
\hline \multirow{3}{*}{ 2. Hostilidad } & 1. Reproche \\
& 2. Insultos \\
& 3. Amenazas \\
\hline \multirow{3}{*}{ 3. Indiferencia } & 1. No empatía ni apoyo \\
\hline \multirow{3}{*}{ 4. Intimidación } & 2. Monopolización \\
& 1. Juzgar, criticar, corregir \\
& 2. Posturas y gestos amenazantes \\
& 3. Conductas destructivas \\
\hline \multirow{5}{*}{ 5. Imposición de conductas } & 1. Bloqueo social \\
& 2. Ordenes \\
& 3. Desviaciones \\
& 4. Insistencia abusiva \\
& 5. Invasiones en la privacidad \\
& 6. Sabotajes \\
\hline
\end{tabular}




\begin{tabular}{ll}
\hline 6. Culpabilización & 1. Acusaciones \\
& $\begin{array}{l}\text { 2. Gaslighting ("Luz de gas") } \\
\text { 3. Negación / desmentida }\end{array}$ \\
\hline 7. Bondad aparente & Manipulación de la realidad \\
\hline
\end{tabular}

\section{Resultados}

Los análisis efectuados fueron los siguientes: en primer lugar, realizamos un análisis descriptivo de cada uno de los factores y subfactores que integran el estudio (desvalorización, hostilidad, indiferencia, intimidación, imposición de conductas, culpabilización y bondad aparente). Con el análisis descriptivo valoramos la presencia de indicadores y/o manifestaciones de maltrato psicológico en la muestra global $(n=216)$, y el nivel de adecuación.

A continuación, realizamos el análisis inferencial con la finalidad de analizar las diferencias significativas entre los grupos de edad considerados (17-18, 19-20, 21-22 y 23 ó más). Para ello, comprobamos en un primer momento si nuestras variables cumplen los requisitos para realizar pruebas paramétricas, utilizando así la prueba de Kolmogorov-Smirnov para demostrar si nuestra distribución es normal dentro de la población, la prueba de Rachas que nos indica si la muestra es aleatoria y la prueba de Levene que presenta si las varianzas son homogéneas. Constatando así que es correcto realizar pruebas paramétricas procedemos a utilizar el ANOVA de un factor.

En relación a los diferentes componentes del maltrato psicológico analizados, los resultados del Cuestionario de Maltrato Emocional concluyen que todos se encuentran afectados, destacando las puntuaciones obtenidas por los sujetos en el factor indiferencia donde el porcentaje global de sujetos cuyo nivel de adecuación es "alto" y "muy alto" alcanza el 37\%.

Como se aprecia en la tabla 4, la presencia de cada uno de los factores relacionados con el maltrato psicológico que tiene lugar en el seno de las parejas evaluadas, se distribuye de forma bastante homogénea entre los sujetos, ya sea desde niveles de presentación moderada como altos y muy altos. Si nos centramos en los valores que reflejan la aparición de cada factor en la pareja con una frecuencia moderada, encontramos que todos ellos se sitúan en un 
intervalo del 47\%-58\% quedando representados los porcentajes límite por el factor bondad aparente $(46,8 \%)$ e indiferencia $(57,9 \%)$. En cuanto a los valores que expresan la aparición de factores de violencia emocional en la pareja con una frecuencia elevada, es decir, representando a los sujetos con con un nivel de adecuación alto y muy alto, destacar el intervalo que comprende desde el 33\%-37\% donde se sitúan todas las puntuaciones obtenidas en esta categoría y cuyos porcentajes límite se registran en los factores hostilidad, imposición de conductas y bondad aparente (33\%) frente al de indiferencia (37\%).

A su vez, es importante señalar que el nivel de ejecución de conductas de indiferencia no presenta importantes diferencias respecto a las manifestaciones de desvalorización, intimidación y culpabilización en estos jóvenes, quienes ponen en práctica este tipo de comportamientos con una frecuencia del 34\%. De esta forma, podemos afirmar que, las conductas de indiferencia son empleadas con una frecuencia mayor que las de desvalorización, intimidación y culpabilización en un 3\%. Asimismo, la puesta en práctica de manifestaciones hostiles en la pareja (hostilidad), de carácter impositivo (imposición de conducta) y de manipulación (bondad aparente) se produce en un 33\%, siendo por tanto, menos frecuentes que las de desvalorización, intimidación y culpabilización aunque en un punto porcentual correspondiente al $1 \%$.

Tabla 4. Sujetos observados en la aplicación del CME según nivel de adecuación

\begin{tabular}{|c|c|c|c|c|}
\hline FACTOR & NIVEL & $\overline{\mathbf{N}}$ & & \\
\hline & $>80:$ muy alto & 21 & 9,7 & \\
\hline Desvalorización & 60-80: alto & 52 & 24,1 & 34 \\
\hline & 40-60: medio & 112 & 51,9 & \\
\hline & $<40$ : bajo & 31 & 14,4 & \\
\hline & $>48:$ muy alto & 21 & 9,7 & \\
\hline Hostilidad & 36-48: alto & 51 & 23,6 & 33 \\
\hline & 24-36: medio & 118 & 54,6 & \\
\hline & $<24$ : bajo & 26 & 12,0 & \\
\hline & $>32:$ muy alto & 21 & 9,7 & \\
\hline Indiferencia & 24-32: alto & 58 & 26,9 & 37 \\
\hline & 16-24: medio & 125 & 57,9 & \\
\hline & $<16$ : bajo & 12 & 5,6 & \\
\hline & $>48:$ muy alto & 21 & 9,7 & \\
\hline Intimidación & 36-48: alto & 53 & 24,5 & 34 \\
\hline & 24-36: medio & 113 & 52,3 & \\
\hline & $<24$ : bajo & 29 & 13,4 & \\
\hline
\end{tabular}




\begin{tabular}{llccc}
\hline \multirow{2}{*}{ Imposición de conductas } & $>97$ : muy alto & 21 & 9,7 & \\
& 72-97: alto & 51 & 23,6 & 33 \\
& 48-72: medio & 124 & 57,4 & \\
& $<48$ : bajo & 20 & 9,3 & \\
\hline \multirow{3}{*}{ Culpabilización } & $>48:$ muy alto & 20 & 9,3 & \\
& 36-48: alto & 53 & 24,5 & 34 \\
& 24-36: medio & 106 & 49,1 & \\
& $<24:$ bajo & 37 & 17,1 & \\
\hline \multirow{3}{*}{ Bondad Aparente } & $>16:$ muy alto & 19 & 8,8 & \\
& 12-16: alto & 53 & 24,5 & 33 \\
& 8-12: medio & 101 & 46,8 & \\
& $<8:$ bajo & 43 & 19,9 & \\
\hline
\end{tabular}

En cuanto a las medias y desviaciones típicas en los diferentes factores de maltrato psicológico, los resultados indican cómo las medias en las puntuaciones de los subfactores del maltrato psicológico son más elevadas en desprecio $(\mathrm{M}=1.97$, DT $=.98)$, insultos $(\mathrm{M}=1.90$, $\mathrm{DT}=.90)$, sabotajes $(\mathrm{M}=1.81, \mathrm{DT}=.90)$ y negación/desmentida $(\mathrm{M}=1.90, \mathrm{DT}=.94)$.

Tabla 5. Medias y desviaciones típicas de los factores del maltrato psicológico

\begin{tabular}{lcc} 
& M & DT \\
\cline { 2 - 3 } I. Desvalorización & 1.71 & .83 \\
Ridiculización & 1.73 & .85 \\
Descalificaciones & 1.66 & .84 \\
Trivializaciones & 1.50 & .80 \\
Oposiciones & 1.55 & .78 \\
Desprecio & 1.97 & .98 \\
\hline II. Hostilidad & 1.69 & .81 \\
Reproche & 1.43 & .74 \\
Insultos & 1.90 & .90 \\
Amenazas & 1.73 & .86 \\
\hline III. Indiferencia & 1.59 & .74 \\
No empatía ni apoyo & 1.55 & .78 \\
Monopolización & 1.64 & .79 \\
\hline IV. Intimidación & 1.69 & .82 \\
Juzgar, criticar, corregir & 1.56 & .77 \\
Posturas y gestos amenazantes & 1.73 & .89 \\
Conductas destructivas & 1.75 & .89 \\
\hline
\end{tabular}




\begin{tabular}{lll}
\hline$V$. Imposición de conductas & 1.66 & .78 \\
Aislamiento social & 1.72 & .86 \\
Órdenes & 1.49 & .81 \\
Desviaciones & 1.65 & .83 \\
Insistencia abusiva & 1.42 & .77 \\
Invasiones en la privacidad & 1.74 & .82 \\
Sabotajes & 1.81 & .90 \\
\hline VI. Culpabilización & 1.74 & .85 \\
Acusaciones & 1.47 & .81 \\
Gaslighting & 1.79 & .88 \\
Negación/desmentida & 1.90 & .94 \\
\hline VII. Bondad aparente & 1.78 & .87 \\
Manipulación de la realidad & 1.79 & .85 \\
\hline
\end{tabular}

A su vez, tal y como podemos evidenciar en la tabla 6 , los resultados indican que los jóvenes con edades comprendidas entre 17-18 años ejercen maltrato emocional poniendo en práctica conductas de desvalorización representadas por los subfactores descalificación, trivialización y oposiciones; hostilidad, con la presencia del subfactor reproche; indiferencia con el subfactor no empatía ni apoyo; imposición con los subfactores ordenes e insistencia abusiva; culpabilización con el subfactor acusaciones y conductas intimidatorias como juzgar, criticar y corregir en sus relaciones de noviazgo. De estas manifestaciones encontramos las puntaciones más altas en las variables: desprecio $(\mathrm{M}=1.74$, $\mathrm{DT}=1.11)$, insultos $(\mathrm{M}=$ $1.71, \mathrm{DT}=.96)$, desviaciones $(\mathrm{M}=1.61, \mathrm{DT}=.97)$, invasiones en la privacidad $(\mathrm{M}=1.61$, $\mathrm{DT}=1.05)$, negación/desmentida $(\mathrm{M}=1.61, \mathrm{DT}=1.05)$ y manipulación de la realidad $(\mathrm{M}=$ 1.61 , DT $=1.00$ ). Por otro lado, podemos observar que desde los 19 a los 22 años las manifestaciones de maltrato que emplean los sujetos son las mismas, concretamente: reproche, ordenes e insistencia abusiva, a excepción de la presencia de trivializaciones en el intervalo que abarca los 19-20 años y de las acusaciones del intervalo de edad que va desde 21-22 años. Los sujetos comprendidos entre los 23 años o más no destacan en ninguna de las manifestaciones analizadas.

En relación a los componentes del maltrato psicológico y el factor edad los resultados obtenidos tras efectuar el ANOVA de un factor, concluyen que existen diferencias en los subfactores oposiciones y ordenes. Estas diferencias vienen marcadas por un nivel de ejecución considerablemente mayor de conductas de oposiciones y ordenes en la pareja por parte de los jóvenes de 17-18 años frente al resto de intervalos de edad, observando que, cuanto más 
avanza el sujeto en la escala evolutiva hacia los 23 años ó más, menos emplea dichas manifestaciones de maltrato psicológico.

La prueba Kolmogorov-Smirnov para dos muestras independientes indica que se constatan diferencias significativas entre los diferentes intervalos de edad en las muestras de sujetos con edades comprendidas entre los 17-18 años y 21-22 años en los subfactores descalificaciones, trivializaciones, conductas destructivas y aislamiento social.

Tabla 6. Medias y desviaciones típicas atendiendo a la edad

\begin{tabular}{|c|c|c|c|c|c|c|c|c|}
\hline & \multicolumn{2}{|c|}{ 17-18 años } & \multicolumn{2}{|c|}{ 19-20 años } & \multicolumn{2}{|c|}{ 21-22 años } & \multicolumn{2}{|c|}{23 ó más } \\
\hline & $\mathbf{M}$ & DT & $\mathbf{M}$ & DT & $\mathbf{M}$ & DT & $\mathbf{M}$ & DT \\
\hline I. Desvalorización & 1.47 & .92 & 1.79 & .83 & 1.71 & .79 & 1.83 & .82 \\
\hline Ridiculización & 1.55 & .95 & 1.79 & .83 & 1.73 & .84 & 1.83 & .76 \\
\hline Descalificaciones & 1.42 & .95 & 1.63 & .80 & 1.73 & .78 & 1.83 & .92 \\
\hline Trivializaciones & 1.29 & .87 & 1.49 & .79 & 1.59 & .78 & 1.54 & .78 \\
\hline Oposiciones & 1.42 & .86 & 1.52 & .77 & 1.52 & .72 & 1.96 & .81 \\
\hline Desprecio & 1.74 & 1.11 & 2.11 & .99 & 1.94 & .91 & 2.00 & .93 \\
\hline II. Hostilidad & 1.47 & .92 & 1.72 & .76 & 1.72 & .79 & 1.83 & .82 \\
\hline Reproche & 1.32 & .87 & 1.42 & .67 & 1.43 & .74 & 1.63 & .77 \\
\hline Insultos & 1.71 & .96 & 1.97 & .92 & 1.92 & .87 & 196 & .81 \\
\hline Amenazas & 1.58 & .98 & 1.79 & .77 & 1.71 & .86 & 1.83 & .92 \\
\hline III. Indiferencia & 1.42 & .86 & 1.65 & .74 & 1.60 & .71 & 1.67 & .64 \\
\hline No empatía ni apoyo & 1.37 & .82 & 1.59 & .75 & 1.53 & .75 & 1.79 & .83 \\
\hline Monopolización & 1.53 & .98 & 1.65 & .79 & 1.67 & .75 & 1,67 & .64 \\
\hline IV. Intimidación & 1.53 & .95 & 1.75 & .81 & 1.67 & .75 & 1.88 & .90 \\
\hline Juzgar, criticar, corregir & 1.47 & .89 & 1.55 & .77 & 1.55 & .68 & 1.79 & .88 \\
\hline Posturas y gestos amenazantes & 1.50 & .98 & 1.80 & .87 & 1.71 & .82 & 1.96 & 1.00 \\
\hline Conductas destructivas & 1.50 & 1.01 & 1.76 & 85 & 1.80 & .84 & 1.92 & .97 \\
\hline V. Imposición de conductas & 1.45 & .89 & 1.72 & .76 & 1.65 & .71 & 1.88 & .85 \\
\hline Aislamiento social & 1.55 & 1.06 & 1.82 & .85 & 1.67 & .75 & 1.83 & .87 \\
\hline Órdenes & 1.29 & .87 & 1.45 & .82 & 1.49 & .72 & 1.88 & .85 \\
\hline Desviaciones & 1.61 & .97 & 1.51 & .75 & 1.73 & .81 & 1.88 & .80 \\
\hline Insistencia abusiva & 1.29 & .92 & 1.49 & .73 & 1,36 & .72 & 1.63 & 77 \\
\hline Invasiones en la privacidad & 1.61 & 1.05 & 1.75 & .79 & 1.76 & .69 & 1.88 & .90 \\
\hline Sabotajes & 1.58 & 1.00 & 1.90 & .91 & 1.82 & .83 & 1.88 & .90 \\
\hline VI. Culpabilización & 1.47 & .92 & 1.82 & .82 & 1.78 & .86 & 1.79 & .78 \\
\hline Acusaciones & 1.29 & .87 & 1.56 & .84 & 1.42 & .72 & 1.67 & .87 \\
\hline Gaslighting & 1.53 & .98 & 1.86 & .85 & 1.82 & .87 & 1.92 & .78 \\
\hline Negación/desmentida & 1.61 & 1.05 & 1.99 & .95 & 1.92 & .90 & 2.08 & .83 \\
\hline VII. Bondad aparente & 1.55 & 1.06 & 1.85 & .87 & 1.80 & .76 & 1.88 & .85 \\
\hline Manipulación de la realidad & 1.61 & 1.00 & 1.86 & .87 & 1.80 & .76 & 1.88 & .85 \\
\hline
\end{tabular}


Tabla 7. ANOVA relativo a la edad y factores del maltrato psicológico

\begin{tabular}{|c|c|}
\hline & $\mathbf{F}$ \\
\hline I. Desvalorización & 1.420 \\
\hline Ridiculización & .786 \\
\hline Descalificaciones & 1.634 \\
\hline Trivializaciones & 1.255 \\
\hline Oposiciones & $2.665^{*}$ \\
\hline Desprecio & 1.255 \\
\hline II. Hostilidad & 1.240 \\
\hline Reproche & .850 \\
\hline Insultos & .756 \\
\hline Amenazas & .627 \\
\hline III. Indiferencia & .893 \\
\hline No empatía ni apoyo & 1.568 \\
\hline Monopolización & .320 \\
\hline IV. Intimidación & 1.023 \\
\hline Juzgar, criticar, corregir & .874 \\
\hline Posturas y gestos amenazantes & 1.554 \\
\hline Conductas destructivas & 1.354 \\
\hline V. Imposición de conductas & 1.708 \\
\hline Aislamiento social & 1.003 \\
\hline Órdenes & $2.723 *$ \\
\hline Desviaciones & 1.643 \\
\hline Insistencia abusiva & 1.297 \\
\hline Invasiones en la privacidad & .576 \\
\hline Sabotajes & 1.132 \\
\hline VI. Culpabilización & 1.548 \\
\hline Acusaciones & 1.538 \\
\hline Gaslighting & 1.498 \\
\hline Negación/desmentida & 1.764 \\
\hline VII. Bondad aparente & 1.112 \\
\hline Manipulación de la realidad & .832 \\
\hline Nota: $* \mathrm{p}<.05 ; * * \mathrm{p}<.01 ; * * * \mathrm{p}<.001$ & \\
\hline
\end{tabular}


Tabla 8. Prueba de Kolmogorov-Smirnov para dos muestras atendiendo a los diferentes intervalos de edad

\begin{tabular}{|c|c|c|c|c|c|c|}
\hline & \\
\hline & $\begin{array}{l}17-18 \\
19-20\end{array}$ & $\begin{array}{l}17-18 \\
21-22\end{array}$ & $\begin{array}{c}\text { 17-18 } \\
23 \text { ó más }\end{array}$ & $\begin{array}{l}19-20 \\
21-22\end{array}$ & $\begin{array}{c}\text { 19-20 } \\
23 \text { ó más }\end{array}$ & $\begin{array}{c}\text { 21-22 } \\
23 \text { ó más }\end{array}$ \\
\hline & $\bar{Z}$ & $\bar{Z}$ & $\overline{\mathbf{Z}}$ & $\mathbf{Z}$ & $\overline{\mathbf{Z}}$ & $\mathbf{Z}$ \\
\hline I. Desvalorización & ,88 & 1,14 & ,64 & ,54 & , 122 & ,483 \\
\hline Ridiculización &, 50 &, 50 & 60 &, 18 & 239 & ,364 \\
\hline Descalificaciones &, 78 & $1,54 *$ & 62 & 90 & ,698 & ,791 \\
\hline Trivializaciones & 68 & $1,57^{*}$ & 45 & 1,06 & 181 &, 830 \\
\hline Oposiciones & 43 &, 40 & 82 &, 53 & 82 & 1,10 \\
\hline Desprecio &, 75 &, 75 & ,45 & 1,11 &, 50 & 27 \\
\hline II. Hostilidad & ,88 & 1,20 & ,64 & 36 & ,40 & ,48 \\
\hline Re0proche &, 50 &, 83 & 66 &, 58 & ,44 & ,43 \\
\hline Insultos & ,88 & 1,00 & ,86 &, 45 & 37 &, 13 \\
\hline Amenazas &, 67 &, 83 & ,34 &, 50 & ,46 &, 58 \\
\hline III. Indiferencia &, 52 & 95 & ,64 & 37 &, 18 &, 30 \\
\hline No empatía ni apoyo &, 45 & 60 & 60 & 23 &, 52 & 69 \\
\hline Monopolización &, 67 & ,89 & 61 &, 51 & 30 & ,39 \\
\hline IV. Intimidación &, 52 & 1,00 & ,51 & ,59 &, 58 & 1,00 \\
\hline Juzgar, criticar, corregir & 60 & 60 & 45 & 21 &, 76 & ,92 \\
\hline Posturas y gestos amenazantes & ,93 &, 88 & ,99 &, 65 &, 81 & 1,30 \\
\hline Conductas destructivas & ,93 & $1,41 *$ & 63 &, 55 &, 75 & ,89 \\
\hline V. Imposición de conductas & ,88 & 1,20 & ,64 &, 55 & ,58 & 97 \\
\hline Aislamiento social & 1,00 & $1,41 *$ & 68 & 1,02 &, 10 &, 82 \\
\hline Órdenes & 42 &, 58 & 84 & ,38 &, 74 & 92 \\
\hline Desviaciones &, 83 & 1,3 & 90 & 1,25 &, 79 & 38 \\
\hline Insistencia abusiva &, 75 &, 59 &, 76 & ,31 & 23 & 43 \\
\hline Invasiones en la privacidad &, 88 & 1,08 & 48 & 27 &, 64 &, 84 \\
\hline Sabotajes &, 87 & 1,03 & ,580 &, 65 & 1,00 &, 37 \\
\hline VI. Culpabilización &, 88 & 1,14 & 639 &, 32 &, 13 & ,29 \\
\hline Acusaciones &, 82 &, 84 & 606 &, 72 & 29 &, 79 \\
\hline Gaslighting &, 88 & 1,20 & ,959 &, 36 &, 31 &, 29 \\
\hline Negación/desmentida &, 80 & 1,14 & 1,12 &, 64 &, 55 & 30 \\
\hline VII. Bondad aparente & ,88 & 1,20 & ,64 & 66 & 12 & 60 \\
\hline Manipulación de la realidad & ,95 & 1,20 & ,64 & 66 &, 12 & 60 \\
\hline
\end{tabular}

\section{Discusión y Conclusiones}

A través de la investigación confirmamos que los jóvenes universitarios con edades comprendidas entre los 17 y 23 años describen patrones de interacción coactiva en sus relaciones de noviazgo. La información arrojada por este estudio, en coherencia con múltiples trabajos sobre violencia prematrimonial (Aguirre y García, 1996; Reyes, 1997; Vizcarra y Poo, 2007) en población donde un 50\% de la muestra se confiesa víctima de alguna agresión 
psicológica (al menos una vez a lo largo de la vida), evidencia la presencia de un elevado número de factores y subfactores que componen el constructo "violencia emocional" en la muestra considerada.

Todo parece indicar que la presencia de cada uno de estos indicadores de violencia psicológica como elementos reguladores de la interacción conyugal puede irrumpir en cualquier momento de la vida en pareja, adquiriendo diferentes formas de expresión que se irán transformando en función de la evolución de la propia pareja (Castellano, García, Lago, Ramírez de Arellano, 1999). De esta forma, aunque partimos de la existencia de propiedades inherentes a la dinámica de maltrato como el carácter cíclico y la intensidad creciente de las agresiones (Walker, 2000), comprobamos la existencia del empleo diferencial de los mismos según la edad en que se encuentra el sujeto, siendo puestos en práctica en mayor medida por los jóvenes de 17-18 años.

No obstante, de acuerdo a la Encuesta Nacional de Victimización Criminal, que afirma que las tasas de violencia íntima aumentan entre los 15 y 19 años alcanzando su máximo entre los 20 y 24 años, siendo la adultez temprana el periodo de la vida con mayor riesgo de violencia (Lewis y Fremouw, 2000; Tolan, Gorman-Smith y Henry, 2006), en nuestra investigación podemos afirmar una mayor evidencia entre los 17-18 años y 21-22, en los subfactores descalificaciones, trivializaciones, conductas destructivas y aislamiento social, clasificados dentro de los factores desvalorización, intimidación e imposición de conductas. Tal y como señala Taverniers (2001), las descalificaciones es la forma de abuso en que típicamente se descarta la parte femenina/masculina de la víctima así como sus habilidades, realidad o experiencia. Es un mecanismo base dirigido a infundar los sentimientos de inferioridad necesarios para que se establezca la relación asimétrica que propicia la aparición de otras manifestaciones violentas en la pareja, ya sea de forma enmascarada o explícita. Este subfactor, queda reflejado según los indicadores referidos en nuestra investigación, en críticas corrosivas, humillaciones y frecuentes conductas de desautorización, que facilitan la puesta en práctica de actitudes muy sutiles que ponen en tela de juicio la relevancia de cada una de las acciones que efectúa el sujeto a diario, mediante la trivialización de las mismas. Los indicadores encontrados de este último subfactor en nuestro trabajo, aluden a comentarios de infravaloración y conductas de no reconocimiento del éxito personal recogido por la pareja. Instaurada esta dinámica relacional cuyo objetivo se centra en establecer continua y sistemáticamente el control sobre la víctima (Loring, 1994) la aparición de conductas destructivas con ánimo de atemorizar a la 
misma y obligarla a mantenerse en el seno de la pareja, es una estrategia frecuente que, a juzgar por los indicadores plasmados en nuestro trabajo se materializa en diversas puestas en escena que van desde la destrucción de objetos de valor económico o afectivo para la víctima hasta el maltrato de sus animales domésticos. El agresor/a alcanzará el control total sobre la víctima cuando le impone conductas de bloqueo social que la aíslan de familiares, amigos y cualquier contacto que ésta pueda entablar con un mundo exterior que no este al alcance de su control y manipulación. Las manifestaciones de este subfactor aquí recogidas se sintetizan en intentos de restricción continua, ya sea, de tipo afectivo o social hasta límites degradantes.

En cuanto a los jóvenes con edades comprendidas entre los 19 a los 22 años podemos señalar un repertorio conductual caracterizado por un elevado número tanto de conductas hostiles como las de reproche como de tipo impositivo como las órdenes e insistencia abusiva. Diferentes autores (Loring, 1994; Taverniers, 2001), identifican el reproche como la forma de hostilidad probablemente más frecuente de la violencia conyugal, erigiéndose en la psicopatología de la vida cotidiana de la pareja. Es entendido como un mecanismo violento, aunque no de agresión explícita, que consiste en exigir al otro de forma rígida y estereotipada, que se adapte a las propias expectativas de cómo ha de ser y actuar pasando por alto la individualidad del mismo. en recriminaciones sobre hechos sucedidos en el pasado, repetidas quejas sobre aspectos que le insatisfacen de su persona, comentarios maliciosos cuyo único objetivo es alterar intencionadamente el bienestar de la pareja y, el más representativo, la constante censura de la conducta llevada a cabo por el sujeto, en virtud de aquella que, arbitrariamente, estima como apropiada.

Pero si el reproche es de una indudable naturaleza acusatoria, que surge de la no aceptación real del otro, hemos de resaltar la coherencia de los resultados obtenidos en este trabajo, destacando el papel de las órdenes, como otro de los subfactores más comúnmente empleados por estos sujetos, que a su vez, nace de la no admisión de igualdad y autonomía de la otra persona. El reflejo de esta realidad queda identificado en nuestra investigación a través de aquellas conductas de imposición directa que demandan inflexiblemente el cumplimiento de las apetencias del agresor/a, en lugar de pedir respetuosamente lo que solicita.

En cuanto a la insistencia abusiva, debemos destacar que, de nuevo, es una forma de imposición de conducta que se caracteriza por reiterar las demandas personales a la pareja hasta obtener de ella los propósitos marcados, a través de generarle cansancio. Los indicado- 
res encontrados de este subfactor aluden a la persistencia del sujeto para obtener la razón ante cualquier situación de discrepancia, inducir a la pareja a realizar prácticas sexuales displacenteras para ésta, presionar al sujeto hasta que éste desista de hacer sus propios planes en virtud de los suyos y, por último, la obstinación implacable del sujeto hasta obtener de la pareja todos sus deseos.

Finalmente, subrayar el carácter extendido de los malos tratos psicológicos cometidos entre jóvenes universitarios que queda reflejado en nuestro estudio y es corroborado por un amplio volumen de investigaciones que así lo ratifican. Tal es el caso de Henton, Cate, Koval, Lloyd y Christopher (1983), donde se arrojaron datos que identificaban la situación de abuso conyugal en 121 de cada 1000 casos y, Matthews (1984) quien, con posterioridad, en una muestra de 351 universitarios, reveló que 79 personas, habían sufrido al menos un incidente de violencia en durante sus relaciones de noviazgo. En esta misma línea, destacamos los trabajos de Deal y Wampler (1986), quienes tan sólo dos años después, corroboraron dichos hallazgos en una muestra de 410 donde el 47\% informó de haber experimentado alguna violencia en sus relaciones de noviazgo. No obstante, en ningún momento precisaron la prevalencia de los diferentes tipos de violencia. Por ello, consideramos de interés señalar la investigación de McKinney (1986) que, sin duda, supuso en este mismo año un ligero avance en el estudio del maltrato emocional durante el noviazgo, poniendo sobre la mesa, a partir de una muestra de 163 universitarios, un porcentaje del 85\% conformado por ambos sexos que declararon ser víctimas de abusos de naturaleza psíquica en sus relaciones de noviazgo. Evidenciamos pues, de acuerdo a los estudios de Dobash y Dobash (1978), Echeburúa (1996), Rosenbaum y O'Leary (1981) y Walker (1979), que varios de los factores y subfactores del maltrato psicológico en la pareja comienzan a manifestarse en las relaciones de noviazgo.

Por último, nos gustaría concluir diciendo que no basta con aportar evidencias empíricas que, como en este caso, constaten la existencia de una violencia emocional, desencadenante de la violencia física, en edades tempranas donde los pilares de la pareja se encuentran en ciernes. Obviamente, consideramos de vital importancia poner en marcha estudios que favorezcan el conocimiento de aspectos como la prevalencia de indicadores y/o manifestaciones de violencia psicológica y los factores de riesgo que favorecen la aparición y el mantenimiento del maltrato psicológico, pero como punto de partida de otras medidas tales a la integración en el ámbito educativo, concretamente en el contexto de la Educación Secundaria (De 
la Fuente, Peralta, Sánchez Roda, 2006) de actuaciones dirigidas a prevenir la conflictividad en las relaciones de pareja.

En este sentido, a pesar de que la inteligencia emocional es un concepto de reciente evolución que ha sido investigado, fundamentalmente, en el ámbito académico y organizacional, si tenemos en cuenta su demostrada efectividad en ambos sectores, aumentando la calidad de vida del alumno (Fernández-Berrocal y Extremera, 2002) y del trabajador (Goleman, 1999), respectivamente, cabe cuestionarse la posible generalización de los mismos al campo de la pareja. De esta forma, condideramos oportuno llevar a cabo la implementación de programas basados en el entrenamiento de competencias comprendidas en la inteligencia emocional que permitan introducir cambios de actitud y comportamientos del alumnado en relación a la pareja. No hay que olvidar que múltiples limitaciones de carácter psicológico y comportamental presentes en la violencia conyugal, se encuentran muy relacionadas con conceptos y componentes fundamentales de la Inteligencia emocional.

El Informe Delors (1996) menciona cuatro bases fundamentales para la educación en el siglo XXI que no refieren la educación conceptual o intelectual en exclusiva: aprender a conocer, aprender a hacer, aprender a convivir y aprender a ser. Sólo así favoreceremos medidas de prevención primaria que capaciten al sujeto para afrontar eficazmente los desafíos que se presentan en la vida cotidiana fomentando la protección de conductas de riesgo y potenciando hábitos saludables en lo relativo a la convivencia en pareja (Blázquez, Moreno y García-Baamonde, 2008; Filella, Soldevila, Cabello, Franco, Morel, Farré, 2008). 


\section{Referencias}

Aguirre, A. M. y García, M. (1996). Violencia Prematrimonial en universitarios de la Quinta Región (Chile). Terapia Psicológica, 26, 11-19.

Asensi, L. (2008). La prueba pericial psicológica en asuntos de violencia de género. Revista Internauta de Práctica Jurídica, 21, 15-29.

Basoglu, M. (2007). A mental health care model for earthquake survivors. En U. Niaz, ed. Pakistan earthquake 2005. The Day the Mountains moved: International Perspectives on Handling Psychotrauma, pp.194-199. SAMA Editorial and Publishing Services.

Bertalanffy, L. (1959). The Theory of Open Systems in Physics and Biology. Science 3, 23-29. Blázquez, M., Moreno, J. M. y García-Baamonde, M. E. (2008). El maltrato psicológico en los modelos teóricos explicativos de la violencia de género. Revista del Instituto de Investigaciones de la Facultad de Psicología/ UBA, 13 (2).

Castellano, I., García, M. J., Lago, M. J. y Ramírez de Arellano, L. (1999). La violencia en parejas universitarias. Boletín Criminológico, 42, 1-4.

Corsi, J. y Ferreira, G. (1998). Manual de capacitación y recursos para la prevención de la violencia familiar. Buenos Aires: Asociación Argentina de Prevención de la Violencia Familiar.

Deal, J. E. y Wampler, K. S. (1986). Dating violence: The primacy of previous experience. Journal of Social and Personal Relationships, 3, 457-471.

De la Fuente, J., Peralta, F. J., Sánchez Roda, M.D. (2006). Valores sociopersonales y problemas de convivencia en la Educación Secundaria. Electronic Journal of Research in Educational Psychology. 4 (2), 171-200.

Delors, J. (1996). La Educación encierra un tesoro. Informa a la UNESCO de la Comisión Internacional sobre la Educación para el siglo XXI. Madrid: Santillana, Ediciones Unesco.

Dobash, R. E, Dobash, R. P. (1979). Violence against wives: A case against the patriarchy. New York: Free Press, Open Books, Shepton Mallet.

Echeburúa, E. (1996). Personalidades violentas. Madrid: Pirámide.

Fernández-Berrocal, P. y Extremera, N. (2002). La Inteligencia Emocional como una habilidad esencial en la escuela. OEI-Revista Iberoamericana de Educación, 29, 1-6.

Filella, G., Soldevila, A., Cabello, E., Franco, L., Morel, A. y Farré, N. (2008). Diseño, aplicación y evaluación de unn programa de educación emocional en un centro penitenciario. Electronic Journal of Research in Educational Psychology. 15, 6 (2), 383-400. 
Follingstad, D. R., Rutledge, L. L., Berg, B.J., Hause, E. S. y Polek, D.S.(1990). The role of emotional abuse in physically abusive relationships. Journal of Family Violence, 5 (2), 107-120.

Garner, J.W. y Hudson, W.W. (1992). Non Psysical abuse of partner scale (NPAPS). Extraído de: http://www.walmyr. com/ NPAPSSAM.pdf el 18 de abril de 2005.

Glass N., Freland, N., Campbell, J., Yonas, M., Sharp, P. y Kub, J. (2003). Adolescent Dating Violence: Prevalence, Risk Factors, Health Out-comes, and Implications for Clinical Practice. Journal of Obstetric, Gynecologic, \& Neonatal Nursing, 32, (2).

Goleman, D. (1999). La inteligencia emocional en la empresa. Buenos Aires: Javier Vergara Editor.

Grumbaum, J.A., Kann, L., Kinchen, S.A., Williams, B., Ross, J.G., Lowry, R., y Kolbe, L. (2002). Youth risk behavior surveillance. Surveillance Summaries, 9, 1-64.

Henning, K R. y Klesges, L.M. (2003). Impact of intimate partner violence on unmet need for mental health care. American Journal of Public Health 93, 1089-1097.

Henton, J., Cate, R., Koval, J., Lloyd, S. y Christopher, S. (1983). Romance and violence in dating relationships. Journal of Family Issues, 4, 467-482.

Hirigoyen, M. F. (2006). Mujeres maltratadas. Barcelona: Paidós.

Hudson, W.W. (1990). Partner Abuse Scale: Non Physical. Tempe, Arizona: Walmyr Publishing Company.

Hudson, W.W. y Mc Intosh S.R. (1981). The Assessment of Spouse Abuse: Two quantifiable dimensions. Journal of Marriage and Family, 11, 873-88.

Hué, C. (1994). Inteligencia Emocional y prevención del maltrato de género. II Jornadas sobre violencia familiar. Universidad de Zaragoza.

Lewis, S. F., y Fremouw, W. (2000). Dating violence: A critical review of the literature. Clinical Psychology Review, 1, 105-127.

Loring, M. T. (1994) Emotional Abuse. New York: Lexington Books.

Marshall, L. (1992). Development of Severity of Violence Against Women Scales. Journal of family violence, 7 (2).

Matthews, W. J. (1984). Violence in college couples. College Student Journal, 18, 150-158.

Matud, M. P. Caballeira, M. y Marrero, R. J. (2001). Validación dfe un inventario de evaluación del maltato a la mujer por su pareja: el APCM. Psicopatología Clínica, Legal y Forense, 1, 5-17. 
McKinney, K. (1986). Measures of verbal, physical and sexual dating violence by gender. Free Inquiry in Creative Sociology, 14, 55-60.

McNamara, J. R. y Broker, D. J. (2000). The abuse disability questionnaire: A new scale for asseseing the consequences of partner abuse. Journal of Interpersonal violence, 15 (2), 170-183.

Montero, A. (2005). Sociedad y violencia machista hacia la mujer. Temas para el debate, 133, 27-30.

O'Leary, K. D. (1988). Physical aggression between spouses: A social learning perspective.In V. B. Van Hasselt, R. L. Morrison, A. S. Bellack, \& M. Hersen (Eds.). Handbook of Family Violence, pp. 31-55. Plenum Press.

Price, L., Byers, S., Sears, H, Whelan, J. Y Saint-Pierre, M. (2000). Dating Violence amongst New Brunswick Adolescents: A Summary of Two Studies. Research Paper Series, 2 (1).

Reiss, A. y Roth, J. (1993). Understanding and preventing violence. Washington, D.C.: National Academy Press.

Reyes P (1997). La violencia psicológica en las relaciones de pololeo o noviazgo entre adultos jóvenes. Santiago: Pontificia Universidad Católica de Chile.

Rosenbaum, A. y O'Leary, D. (1981). Marital violence: characteristics of abusive couples. Journal of Counsulting and Clinical Psychology, 49 (1), 63-71.

Sackett, L. A y Saunders, D. G. (1999). The impact of different forms of psychological abuse on battered women. Violence and Victims, 14, 105-117.

Salazar, T., Torres, I. y Rincón, V. (2005). Violencia en la pareja. Capítulo Criminológico ,33 (1).

Smith, L.J.F. (1993). Domestic Violence: an overview ot the literature. Londres: HMSO.

Sonkin, D.J. (2001). Domestic violence: The court mandated perpetrator assessment and treatment handbook. Sausalito, CA: Self published by Daniel Sonkin, Ph.D.

Straus, M.A. (1979). Measuring intrafamily conflict and aggression: The Conflict Tactics Scale (CTS). Journal of Marriage and The Family, 41, 75-88.

Straus, M.A. (1983). Ordinary violence, child abuse, and wife-beating. In D. Finkelhor, R.J. Gelles, G.T. Hotaling y M.A. Straus (Eds.), The dark side of families: Current family violence research, pp. 213-234. Newbury Park, California: Sage.

Straus, M.A., Hamby, S.L., Boney-McCoy, S., Sugarman, D.B. (1996). The revised Conflict Tactics Scales (CTS2): development and preliminary psychometric data. Journal of Family Issues, 17(3): 283-316. 
Street A. y Arias I. (2001). Psychological abuse and postraumatic stress disorder in battered women: examining the roles of shame and guilty. Violence Victims, $16(1), 65-78$.

Sugarman, D. y Hotaling, G. (1989). Violencia en la pareja: prevalencia, contexto y calificadores de riesgo. En M. Pirog-Good y J. Stets, (eds.). Violence in dating relationships: Emerging Social Issues, pp. 3-32. New York: Praeger.

Swahnberg, I. M. \& Wijma, B. (2003). The NorVold Abuse Questionnaire (NorAQ): validation of new measures of emotional, physical, and sexual abuse, and abuse in the health care system among women. European Journal of Public Health, 13, 361-366.

Taverniers, K. (2001). Abuso emocional en parejas heterosexuales. Revista Argentina de Sexualidad Humana, 15 (1), 28-34.

Tolan, P, Gorman-Smith, D. y Henry D. (2006). Domestic Violence Prevalence Family Violence, Annual Review of Psychology 57, 557-583.

Tolman, R. M. (1989). The development of a measure of psychological maltreatment of women by their male partners. Violence and Victims, 4, 159-177.

Vizcarra B. y Poo, A. M. (2007). Violencia en jóvenes universitarios: Una realidad silenciada. Temuco (Chile): Dirección de Investigación Universidad de La Frontera.

Walker L. E. (1979). The battered woman. New York: Harper and Row.

Walker, L.E. (2000). The battered woman syndrome. New York: Springer Publishing Company. 